\title{
Application of local gauge theories to fluid mechanics
}

\author{
Thomas Merz ${ }^{1} \dagger$ \\ ${ }^{1} 8055$ Zürich, CH
}

The problem of fluid dynamics can be greatly simplified if, for every point in space, the strain-rate tensor is diagonalized. This tensor is introduced into the Navier-Stokes equations via constitutive equation and divergence of the stress tensor. This article shows that local $S O(3) \times U(1)$ gauge fields can be used to locally diagonalize the diffusion components of the strain-rate tensor. The gauge fields resulting from the connection can be interpreted as convection components of the flow, they show properties of quasiparticles and can be understood as elementary vortices. Thus, the proposed approach not only offers new insights for the solution and situative simplification of the Navier-Stokes equations, it also uncovers hidden symmetries within the flow convection, allowing - depending on boundary conditions - further physical interpretation.

Keywords: fluid dynamics; turbulent flow; stationary flow; $\mathrm{SO}(3)$ local gauge; quasiparticle; nonlinearity

\section{Introduction}

\subsection{Notation}

In the following, co- and contravariant vectors and second order tensors are written in bold; latin indexes run from 1 to 3, unless otherwise noted. The separation of second order tensors $\mathbf{X} \in \mathbb{R}^{3 \times 3}$ into isotropic, scalar parts $\mathbf{X}_{\|}$and deviatory, trace-free parts $\mathbf{X}_{\perp}$ is
carried out as follows:

$$
\mathbf{X}=\mathbf{X}_{\|}+\mathbf{X}_{\perp} \quad \mathbf{X}_{\|}=\frac{1}{3} \operatorname{tr}(\mathbf{X}) \mathbb{1} \quad \mathbf{X}_{\perp}=\mathbf{X}-\mathbf{X}_{\|} \quad \operatorname{tr}\left(\mathbf{X}_{\perp}\right)=0
$$

for tensors acting on three-dimensional space $\mathbb{R}^{3}$. All quantities used are considered to be dimensionless. In order to keep the notation concise, the customary star ( $)^{*}$ in fluid dynamics is omitted for such quantities. In removing the dimensions, the quantities and standard formulas commonly found in fluid mechanics are used: characteristic length $L$, characteristic velocity $v_{\infty}$ and characteristic density $\rho_{\infty}$ (see e.g. Horner (2011), Borghini (2013), Urbanek (2012)). The dimensionless quantities used are derived from the quantities with dimensions (marked with an ()$\left.^{x}\right)$ as follows:

$$
\rho=\frac{\rho^{x}}{\rho_{\infty}} \quad \mathbf{v}=\frac{\mathbf{v}^{x}}{v_{\infty}} \quad \mathbf{p}=\frac{\mathbf{p}^{x}}{\rho_{\infty} v_{\infty}^{2}} \quad \nabla=L \nabla^{x} \quad t=\frac{v_{\infty} t^{x}}{L} \quad \mathbf{g}=\frac{L \mathbf{g}^{x}}{v_{\infty}^{2}} .
$$

And in the case of the dimensionless viscosities which are functions of the Reynolds number $R e$, i.e. the kinematic viscosity $\nu$, the dynamic viscosity $\mu$ and the volume viscosity $\zeta$ :

$$
R e^{-1}=\nu=\frac{\nu^{x}}{L v_{\infty}} \quad \mu=\operatorname{Re}^{-1} \rho \quad \zeta=\frac{\zeta^{x}}{\mu^{x}} R e^{-1} \rho .
$$

$\dagger$ Email address for correspondence: t.merz@alumni.ethz.ch 


\subsection{Basics}

The motion of viscous fluids is described by the Navier-Stokes equations. These are a special form of the Cauchy momentum equations, which are given by (e.g. Coleman $(2010))$ :

$$
\rho \frac{D \mathbf{v}}{D t}=\rho \frac{\partial \mathbf{v}}{\partial t}+\rho(\mathbf{v} \cdot \nabla) \mathbf{v}=\nabla \cdot \boldsymbol{\sigma}-\nabla p+\rho \mathbf{g} .
$$

$\frac{D \mathbf{v}}{D t}=\frac{\partial \mathbf{v}}{\partial t}+(\mathbf{v} \cdot \boldsymbol{\nabla}) \mathbf{v}$ is sometimes referred to as the material derivative. The specific material properties are contained in the constitutive equation, which in the case of the Navier-Stokes equations is defined by the linear isotropic relationship between the strainrate tensor $\dot{\varepsilon}$ and the Cauchy stress tensor $\boldsymbol{\sigma}$ :

$$
\boldsymbol{\sigma}=2 \mu \dot{\varepsilon}_{\perp}+3 \zeta \dot{\varepsilon}_{\|}=R e^{-1} \rho\left(2 \dot{\varepsilon}_{\perp}+\frac{3 \zeta^{x}}{\mu^{x}} \dot{\varepsilon}_{\|}\right) .
$$

The linearized strain-rate tensor $\dot{\varepsilon}$ is defined as the symmetrical component of the deformation-rate gradient $\mathbf{F}$, given by the dyadic product $\mathbf{F}=(\boldsymbol{\nabla} \otimes \mathbf{v})=\boldsymbol{\nabla} \mathbf{v}^{\mathbf{T}}$, whereby its components are usually written as:

$$
\dot{\varepsilon}^{i}{ }_{j}=\frac{1}{2}\left(\partial^{i} v_{j}+\partial^{j} v_{i}\right) .
$$

Inserting the constitutive equation Eq. 1.5 into the Cauchy momentum equations Eq. 1.4 using definition Eq. 1.6 yields the Navier-Stokes equations in their convective form:

$$
\rho \frac{\partial \mathbf{v}}{\partial t}+\rho(\mathbf{v} \cdot \boldsymbol{\nabla}) \mathbf{v}=\mu(\boldsymbol{\nabla} \cdot \boldsymbol{\nabla}) \mathbf{v}+\frac{1}{3} \mu \boldsymbol{\nabla}(\boldsymbol{\nabla} \cdot \mathbf{v})-\nabla \bar{p}+\rho \mathbf{g} .
$$

Where the scalar components of the stress tensor are contained in the mechanical pressure:

$$
\bar{p}=p-\zeta \boldsymbol{\nabla} \cdot \mathbf{v}
$$

In the following only the stationary Navier-Stokes equations with $\frac{\partial \mathbf{v}}{\partial t}=\frac{\partial \rho}{\partial t}=0$ will be considered. The system of equations also includes the continuity equation, which in the stationary case is reduced to:

$$
\nabla(\rho \mathbf{v})=\nabla(\mathbf{j})=0 .
$$

This defines the mass flow density $\mathbf{j}=\rho \mathbf{v}$.

The continuity equation allows the stationary, dimensionless Navier-Stokes equation to be written in its conservation form:

$$
\boldsymbol{\nabla} \cdot(\rho \mathbf{v} \otimes \mathbf{v})=\mu(\boldsymbol{\nabla} \cdot \boldsymbol{\nabla}) \mathbf{v}+\frac{1}{3} \mu \boldsymbol{\nabla}(\boldsymbol{\nabla} \cdot \mathbf{v})-\nabla \bar{p}+\rho \mathbf{g} .
$$

Previous attempts to use local gauge theory and/ or Euler-Lagrange approaches on fluid mechanics can be found e.g. in Sulaiman \& Handoko (2005), Moulden (2012), E \& Liu (2003), Constantin (2000). The procedure presented in this article differs significantly from these approaches. Here, $S O(3) \times U(1)$ fields are used that couple eventually diffusion and convection while providing new theoretical insights.

\section{Formulation as local gauge theory}

\subsection{Diagonalization of the strain-rate tensor by local rotations}

The strain-rate tensor is symmetric by definition and can therefore, in situations where the flow is uniform $(\dot{\varepsilon} \neq \dot{\varepsilon}(\mathbf{r}))$, be diagonalized with $\dot{\varepsilon}_{\text {diag }}=\mathbf{R}^{\mathrm{T}} \dot{\varepsilon} \mathbf{R}$, where $\mathbf{R} \in S O(3)$ 
is a rotation. One can therefore write:

$$
\dot{\varepsilon}_{\text {diag }}=\left(\begin{array}{ccc}
\partial^{1} v_{1} & 0 & 0 \\
0 & \partial^{2} v_{2} & 0 \\
0 & 0 & \partial^{3} v_{3}
\end{array}\right) .
$$

However, if the strain-rate tensor depends on the location $(\dot{\varepsilon}=\dot{\varepsilon}(\mathbf{r}))$, no general, global rotation can be found that diagonalizes the strain-rate tensor in all space. In order to be able to diagonalize the tensor in every point, the global rotation $\mathbf{R}$ is replaced by a local rotation $\mathbf{R}(\mathbf{r})$. This corresponds to the introduction of a local $S O(3)$ gauge field with $\dot{\varepsilon}_{\text {diag }}(\mathbf{r})=\mathbf{R}^{\mathbf{T}}(\mathbf{r}) \dot{\varepsilon}(\mathbf{r}) \mathbf{R}(\mathbf{r})$. The rotation field thus generated no longer commutes with the derivative in the strain-rate tensor, since now $\boldsymbol{\partial}(\mathbf{v} \mathbf{R}) \neq(\boldsymbol{\partial} \mathbf{v}) \mathbf{R}$.

The $S O(3)$ gauge field can be described as a Yang-Mills theory, making use of its extensively studied theoretical framework. However, the following constraints must be met first:

- The expression must be reduced to a scalar quantity, since Yang-Mills theories are applied to scalar quantities (usually Lagrange densities).

- By the symmetrization according to Eq. 1.6 two derivatives are formed in $\dot{\varepsilon}$, each generating different, non-abelian and self-interacting gauge fields, whose interactions are unclear. The expression should be reduced to a single derivative.

These two points will be discussed in more detail in the next two sections before formulating the theory:

\subsection{Scalar expression via trace}

A simple way to obtain a scalar expression from the tensor $\dot{\varepsilon}_{\text {diag }}(\mathbf{r})$ is via its trace. The trace can be understood as a projection of the tensor onto a global orthonormal basis $\left\{\mathbf{e}_{\mathbf{i}}\right\}$ :

$$
\operatorname{tr}\left(\dot{\varepsilon}_{\operatorname{diag}}(\mathbf{r})\right)=\sum_{i} \mathbf{e}_{\mathbf{i}} \dot{\varepsilon}_{\operatorname{diag}}(\mathbf{r}) \mathbf{e}^{\mathbf{i}}=\sum_{i} \mathbf{e}_{\mathbf{i}} \mathbf{R}^{\mathbf{T}}(\mathbf{r}) \dot{\varepsilon}(\mathbf{r}) \mathbf{R}(\mathbf{r}) \mathbf{e}^{\mathbf{i}}=\sum_{i} \mathbf{O}_{\mathbf{i}}^{\mathbf{T}}(\mathbf{r}) \dot{\varepsilon}(\mathbf{r}) \mathbf{O}^{\mathbf{i}}(\mathbf{r}) .
$$

In the last step the projection on the base components together with the rotation were combined to $\mathbf{O}^{\mathbf{i}}(\mathbf{r})=\mathbf{R}(\mathbf{r}) \mathbf{e}^{\mathbf{i}}$. The $\mathbf{O}^{\mathbf{i}}$ thus transform like vectors $\mathbf{O}^{\mathbf{i}}=\mathbf{R}^{\prime} \mathbf{O}^{\mathbf{i}}$ with respect to global changes of basis.

\subsection{Symmetrization via phase}

To symmetrize the deformation-rate gradient $\mathbf{F}=(\boldsymbol{\nabla} \otimes \mathbf{v})=\boldsymbol{\nabla} \mathbf{v}^{\mathbf{T}}$, the polar decomposition $\mathbf{F}=\mathbf{S P}$ is used, where in general $\mathbf{S}$ is a hermitian matrix and $\mathbf{P}$ is a unitary transformation. When multiplying by its transpose from the right, the unitary components vanish:

$$
\mathbf{F F} \mathbf{F}^{\mathrm{T}}=\mathbf{S P P}^{\mathrm{T}} \mathbf{S}^{\mathrm{T}}=\mathbf{S S}^{\mathrm{T}} .
$$

In the situation considered, the resulting matrix $\mathbf{S}$ is not only hermitian, but symmetrical. Therefore, $\mathbf{S S}^{\mathbf{T}}$ is also symmetric and can be diagonalized by applying a rotational matrix $\mathbf{R}:\left(\mathbf{S S}^{\mathrm{T}}\right)_{\text {diag }}=\mathbf{R}^{\mathrm{T}} \mathbf{S S}^{\mathrm{T}} \mathbf{R}$. Because of Eq. 2.3, $\mathbf{F F}^{\mathrm{T}}$ is then also diagonalized:

$$
(\mathbf{F F})_{\text {diag }}=\mathbf{R}^{\mathrm{T}} \mathbf{F} \mathbf{F}^{\mathrm{T}} \mathbf{R}=\mathbf{R}^{\mathrm{T}} \mathbf{S} \mathbf{S}^{\mathrm{T}} \mathbf{R}=\left(\mathbf{S S}^{\mathrm{T}}\right)_{\mathrm{diag}}
$$

and the squares of the eigenvalues of $\mathbf{F}$ and $\mathbf{S}$ are the same $\lambda_{S, j}^{2}=\lambda_{F, j}^{2}$. Since $\mathbf{S}$ is symmetrical, its eigenvalues are real $\lambda_{S, j} \in \mathbb{R}$, and $\mathbf{S}$ is diagonal as well:

$$
\mathbf{S}_{\text {diag }}=\mathbf{R}^{\mathrm{T}} \mathbf{S R}
$$


On the other hand, the eigenvalues of $\mathbf{F}$ can be expressed as $\lambda_{F, j}=\lambda_{S, j} e^{i \theta_{j}}$, which fulfill $\lambda_{F, j}^{2}=\lambda_{S, j}^{2}$ and whose complex values contain the rotational components. Thus, $\mathbf{F}$ is diagonalized using the same set of eigenvectors as $\mathbf{S}$ :

$$
\mathbf{F}_{\text {diag }}=\mathbf{R}^{\mathbf{T}} \mathbf{F R}=\left(\begin{array}{ccc}
\lambda_{S, 1} e^{i \theta_{1}} & 0 & 0 \\
0 & \lambda_{S, 2} e^{i \theta_{2}} & 0 \\
0 & 0 & \lambda_{S, 3} e^{i \theta_{3}}
\end{array}\right) .
$$

Therefore, because of the initial polar decomposition $\mathbf{F}=\mathbf{S P}$, it is found, that $\mathbf{P}$ is diagonalized in the same configuration as well:

$$
\mathbf{F}_{\text {diag }}=\mathbf{R}^{\mathrm{T}} \mathbf{F R}=\mathbf{R}^{\mathrm{T}} \mathbf{S P R}=\mathbf{R}^{\mathrm{T}} \mathbf{S R} \mathbf{R}^{\mathrm{T}} \mathbf{P R}=\mathbf{S}_{\text {diag }} \mathbf{P}_{\text {diag }}
$$

and since $\mathbf{P}$ is unitary, it contains the phases:

$$
\mathbf{P}_{\text {diag }}=\left(\begin{array}{ccc}
e^{i \theta_{1}} & 0 & 0 \\
0 & e^{i \theta_{2}} & 0 \\
0 & 0 & e^{i \theta_{3}}
\end{array}\right)
$$

Solving for the symmetric part $\mathbf{S}_{\text {diag }}$ and identifying it with the strain-rate tensor $\dot{\varepsilon}_{\text {diag }}$, it can be seen that:

$$
\mathbf{S}_{\text {diag }}=\dot{\varepsilon}_{\text {diag }}=\mathbf{F}_{\text {diag }} \mathbf{P}_{\text {diag }}^{\dagger}=\left(\mathbf{R}^{\mathrm{T}} \mathbf{F R}\right) \mathbf{P}_{\text {diag }}^{\dagger}=\left(\mathbf{R}^{\mathbf{T}}\left(\nabla \mathbf{v}^{\dagger}\right) \mathbf{R}\right) \mathbf{P}_{\text {diag }}^{\dagger} .
$$

Where the values $F^{i}{ }_{j}=\partial^{i} v_{j} \in \mathbb{C}$ can be complex.

The advantage of this approach becomes clear when formulating the interaction terms of the local gauge field in the following section. When using the standard symmetrization approach with $\dot{\varepsilon}^{i}{ }_{j}=\frac{1}{2}\left(\partial^{i} v_{j}+\partial^{j} v_{i}\right)$, the second derivative leads to three additional non-abelian $S O(3)$ gauge fields with self-interaction, whereas the polar decomposition approach $\mathbf{P}_{\operatorname{diag}}(\mathbf{r})=\operatorname{diag}\left(e^{i \theta_{1}(\mathbf{r})}, e^{i \theta_{2}(\mathbf{r})}, e^{i \theta_{3}(\mathbf{r})}\right)$ creates at most three abelian $U(1)$ gauge fields without self-interaction, which can be treated independently.

\subsection{Gauge invariant strain-rate tensor}

In summary, these considerations lead to the following expression for the trace of the strain-rate tensor:

$$
\begin{aligned}
\operatorname{tr}\left[\dot{\varepsilon}_{\text {diag }}\right] & =\operatorname{tr}\left[\mathbf{R}^{\mathrm{T}} \dot{\varepsilon} \mathbf{R}\right] \\
& =\operatorname{tr}\left[\left(\mathbf{R}^{\mathbf{T}}\left(\boldsymbol{\nabla} \mathbf{v}^{\dagger}\right) \mathbf{R}\right) \mathbf{P}_{\text {diag }}^{\dagger}\right] \\
& =\operatorname{tr}\left[\left(\mathbf{R}^{\mathbf{T}} \boldsymbol{\nabla}\right)\left(\mathbf{v}^{\dagger} \mathbf{R} \mathbf{P}_{\text {diag }}^{\dagger}\right)\right]
\end{aligned}
$$

As expected, this expression has a form on which Yang-Mills theory can be applied in order to diagonalize the strain-rate tensor $\dot{\varepsilon}$ everywhere.

For this purpose, the rotation $\mathbf{R}=\mathbf{R}(\mathbf{r})$ and the phase $\mathbf{P}_{\text {diag }}^{\dagger}=\mathbf{P}_{\text {diag }}^{\dagger}(\mathbf{r})$ are defined as position dependent, local rotation and phase fields. In doing so, it must be guaranteed that the expression obtained from local symmetrization and diagonalization describes the same physics as it did before. It must therefore remain invariant under the corresponding localized $S O(3)$ and $U(1)$ transformations. Their influence and interaction on the fields are considered in the following:

\subsubsection{Gauge fields via local rotations}

At first, pure rotations $\mathbf{R}(\mathbf{r})$ with the phase angles set to zero $\left(\theta_{i}=0\right)$ are considered, such that $\mathbf{P}_{\text {diag }}^{\dagger}=\mathbb{1}$ :

$$
\operatorname{tr}\left[\dot{\varepsilon}_{\text {diag }}(\mathbf{r})\right]=\operatorname{tr}\left[\mathbf{R}^{\mathbf{T}}(\mathbf{r}) \boldsymbol{\nabla}\left(\mathbf{v}^{\dagger}(\mathbf{r}) \mathbf{R}(\mathbf{r})\right)\right] .
$$




\section{Application of local gauge theories to fluid mechanics}

As a prerequisite, it is noted that for global rotations, the trace is left invariant under similarity transformations $\operatorname{tr}\left[\mathbf{R}^{\mathrm{T}} \dot{\varepsilon} \mathbf{R}\right]=\operatorname{tr}[\dot{\varepsilon}]$, and the such applied rotations form a global symmetry of this expression. This global symmetry can now be promoted to a local symmetry.

The rotations are represented as exponential functions:

$$
\mathbf{R}(\mathbf{r})=e^{i \sum_{a} \phi_{a}(\mathbf{r}) \mathbf{L}^{\mathbf{a}}}
$$

where the generators are given by the Lie Algebra so(3):

$$
\mathbf{L}^{\mathbf{1}}=-\frac{i}{2}\left(\begin{array}{ccc}
0 & 0 & 0 \\
0 & 0 & -1 \\
0 & 1 & 0
\end{array}\right) \quad \mathbf{L}^{\mathbf{2}}=-\frac{i}{2}\left(\begin{array}{ccc}
0 & 0 & 1 \\
0 & 0 & 0 \\
-1 & 0 & 0
\end{array}\right) \quad \mathbf{L}^{\mathbf{3}}=-\frac{i}{2}\left(\begin{array}{ccc}
0 & -1 & 0 \\
1 & 0 & 0 \\
0 & 0 & 0
\end{array}\right)
$$

The generators are defined such that they fulfill the standard requirements regarding commutator and normalization for Yang Mills theories (e.g. Iliopoulos (2012)):

$$
\left[\mathbf{L}^{\mathbf{a}} \mathbf{L}^{\mathbf{b}}\right]=i f^{a b c} \mathbf{L}^{\mathbf{c}} \quad f^{a b c}=-\frac{1}{2} \varepsilon^{a b c} \quad \operatorname{tr}\left(\mathbf{L}^{\mathbf{a}} \mathbf{L}^{\mathbf{b}}\right)=\frac{1}{2} \delta^{a b},
$$

where $\varepsilon^{a b c}$ is the Levi-Civita symbol and $f^{a b c}$ is the structure constant defined from it.

It is now necessary to determine the local dependence of the rotations. By using $(\mathbf{a} \otimes \mathbf{b})=\mathbf{a b}^{\dagger}$ and switching to component notation, the trace becomes (the position dependency is no longer written explicitly to allow for a compact notation):

$$
\begin{aligned}
\left(\dot{\varepsilon}_{\text {diag }}\right)_{j}^{j} & =R^{T j}{ }_{k} \partial^{k}\left(v_{l}^{\dagger} R_{j}^{l}\right) \\
& =R^{T j}{ }_{k} \partial^{k}\left(v_{l}^{\dagger}\right) R_{j}^{l}+R^{T j}{ }_{k} v_{l}^{\dagger} \partial^{k}\left(R_{j}^{l}\right) \\
& =R^{T j}{ }_{k} \partial^{k}\left(v_{l}^{\dagger}\right) R_{j}^{l}+R_{k}^{T j} v_{l}^{\dagger}\left(\sum_{a} i \partial^{k} \phi_{a}(\mathbf{r}) \mathbf{L}^{\mathbf{a}}\right)^{k} R_{j}^{l} \\
& =R^{T}{ }_{k} \partial^{k}\left(v_{l}^{\dagger}\right) R_{j}^{l}+R_{k}{ }_{k}\left(\sum_{a} i \partial^{k} \phi_{a}(\mathbf{r}) \mathbf{L}^{\mathbf{a}}\right)^{k} v_{l}^{\dagger} R_{j}^{l} \\
& =R^{T j}{ }_{k} \partial^{k}\left(v_{l}^{\dagger}\right) R_{j}^{l}+i R^{T}{ }_{k} A_{\perp}^{k} v_{l}^{\dagger} R^{l}{ }_{j} \\
& =\left(\dot{\varepsilon}_{\text {local }}\right)^{j}{ }_{j}+\left(\dot{\varepsilon}_{\text {connect. SO }(3)}\right)_{j}{ }_{j}
\end{aligned}
$$

where:

$$
A_{\perp}^{k}=\sum_{a}\left(A_{\perp a}\right)^{k} \mathbf{L}^{\mathbf{a}} \quad \text { with } \quad\left(A_{\perp a}\right)^{k}=\partial^{k} \phi_{a}(\mathbf{r}) .
$$

In vectorial notation this reads:

$$
\begin{aligned}
\operatorname{tr}\left[\dot{\varepsilon}_{\text {diag }}(\mathbf{r})\right] & =\operatorname{tr}\left[\mathbf{R}^{\mathbf{T}} \boldsymbol{\nabla}\left(\mathbf{v}^{\dagger}\right) \mathbf{R}\right]+\operatorname{tr}\left[i \mathbf{R}^{\mathbf{T}} \mathbf{A}_{\perp} \mathbf{v}^{\dagger} \mathbf{R}\right] \\
& =\operatorname{tr}\left[\boldsymbol{\nabla} \mathbf{v}^{\dagger}\right]+\operatorname{tr}\left[i \mathbf{A}_{\perp} \mathbf{v}^{\dagger}\right] \\
& =\operatorname{tr}\left[\dot{\varepsilon}_{\text {local }}(\mathbf{r})\right]+\operatorname{tr}\left[\dot{\varepsilon}_{\text {connect. } \mathbf{S O}(\mathbf{3})}(\mathbf{r})\right] .
\end{aligned}
$$

It was used that the trace remains invariant under similarity transformations. By reordering the equation, one sees that the local component can be divided into a diagonal component $\left(\operatorname{tr}\left[\dot{\varepsilon}_{\operatorname{diag}}(\mathbf{r})\right]\right)$ and a Yang-Mills like component which is generated by the connection:

$$
\operatorname{tr}\left[\dot{\varepsilon}_{\text {local }}(\mathbf{r})\right]=\operatorname{tr}\left[\dot{\varepsilon}_{\text {diag }}(\mathbf{r})\right]-\operatorname{tr}\left[\dot{\varepsilon}_{\text {connect. SO(3) }}(\mathbf{r})\right] .
$$

Finally, the rotations commute with the homogeneous, scalar component $\mathbf{X}_{\|}$of tensors 
in general and couple only to the deviatoric, trace-free parts $\mathbf{X}_{\perp}$ :

$$
\begin{aligned}
\mathbf{X}_{\text {diag }} & =\mathbf{R}^{\mathbf{T}}\left(\mathbf{X}_{\perp}+\mathbf{X}_{\|}\right) \mathbf{R} \\
& =\mathbf{R}^{\mathbf{T}} \mathbf{X}_{\perp} \mathbf{R}+\mathbf{X}_{\|} .
\end{aligned}
$$

And hence in the considered case, it holds:

$$
\operatorname{tr}\left[\dot{\varepsilon}_{\perp \text { local }}(\mathbf{r})\right]=\operatorname{tr}\left[\dot{\varepsilon}_{\perp \text { diag }}(\mathbf{r})\right]-\operatorname{tr}\left[\dot{\varepsilon}_{\perp \text { connect. } \mathbf{S O}(3)}(\mathbf{r})\right] .
$$

\subsubsection{Gauge fields via local phase}

The procedure for the local phase field $\mathbf{P}_{\text {diag }}^{\dagger}(\mathbf{r})$ is essentially the same. What needs to be examined additionally is the interaction between the real rotations $\mathbf{R}(\mathbf{r})$ and the phase field. Therefore, two cases are distinguished:

Case 1: without real rotations. Let all rotation angles be equal to zero $\left(\phi_{a}=0\right)$ and thus $\mathbf{R}(\mathbf{r})=\mathbb{1}$.

Then by definition the deformation-rate gradient already is diagonalized $F^{j}{ }_{k}=\delta_{k}^{j} \partial^{j} v_{k}^{\dagger}$. The velocity components can be expressed as a magnitude and phase: $v_{k}^{\dagger}=\left|v_{k}\right| e^{i \theta_{k}}=$ $v_{\mathbb{R}, k} e^{i \theta_{k}}$, and the components of the complex rotation can be reduced directly in the initial strain-rate tensor from Eq. 2.10.

$$
\boldsymbol{\nabla}\left(\mathbf{v}^{\dagger} \mathbf{P}_{\text {diag }}^{\dagger}\right)=\nabla\left(\mathbf{v}_{\mathbb{R}} \mathbf{P}_{\text {diag }} \mathbf{P}_{\text {diag }}^{\dagger}\right)=\nabla \mathbf{v}_{\mathbb{R}}
$$

This is also the expected result, since the eigenvalues of the strain-rate tensor are directly visible in its diagonal shape, and they must be real in a symmetrical tensor.

The problem has thus already been solved, without necessity to specifically consider the position dependency of the phase.

Case 2: with real rotations. As soon as real rotations $\mathbf{R}$ are introduced into the equation, the procedure above will no longer work.

The complex rotations $\mathbf{P}_{\text {diag }}^{\dagger} \in P$ with $P=\left\{\mathbf{P} \in G L(3, \mathbb{C})\left|\mathbf{P}^{\dagger} \mathbf{P}=\mathbb{1},\right| \operatorname{det} \mathbf{P} \mid=1\right.$, $\left.P^{i}{ }_{j}=\delta_{j}^{i} \lambda_{j}\right\}$ need to be taken into account as local gauge fields. To furthermore get the most efficient approach, it must be ensured that only those complex rotations are applied, which do not interact with the already considered real rotations, i.e. that are acting orthogonal to the real rotations.

To find this orthogonal part, the following observation about the symmetries of the problem is made: The solution space is the space of the $3 \times 3$ diagonal matrices with real eigenvalues $\left(\lambda_{1}, \lambda_{2}, \lambda_{3} \in \mathbb{R}\right)$ and thus has dimension three $\left(d_{\text {solution }}=3\right)$.

The group action of the previously applied real rotations $S O(3)=\{\mathbf{R} \in G L(3, \mathbb{R}) \mid$ $\mathbf{R}^{\mathbf{T}} \mathbf{R}=\mathbb{1}$, $\left.\operatorname{det} \mathbf{R}=1\right\}$ on the $\mathbb{R}^{3}$ is isomorphic to the unit sphere $S^{2}$ and spans a twodimensional sub-vector space $\left(d_{S O(3)}=2\right)$.

Thus, after applying the real rotations, the complementary space that was not considered yet has dimension one: $d_{\text {compl }}=d_{\text {solution }}-d_{S O(3)}=1$, meaning that the complex rotations orthogonal to the real rotations must span a one-dimensional sub-vector space.

A one-dimensional vector space of complex rotations is isomorphic to a phase $U(1)$, and therefore $\mathbf{P}=e^{i \theta}$ can be chosen. However, this description of a complex rotation only works in a properly chosen coordinate system. Hence, the subspace orthogonal to the real rotations has to be found explicitly and expressed correctly to apply the complex rotations as a phase. The existence of this subspace can be expressed as follows:

Both $S O(3)$ and $U(1)$ are subgroups of the unitary $3 \times 3$ matrices $U(3)=\{\mathbf{U} \in$ $\left.G L(3, \mathbb{C})\left|\mathbf{U}^{\dagger} \mathbf{U}=\mathbb{1},\right| \operatorname{det} \mathbf{U} \mid=1\right\} . U(1)$ is the normal subgroup of $U(3)$, defined as the subgroup that commutes with each element of the group. Therefore, $U(1) \rtimes S O(3)$ forms a semidirect product, the two subgroups have a trivial intersection $U(1) \cap S O(3)=\mathbb{1}$, and $U(1)$ is again the normal subgroup of this composite group. 
After showing that the searched subspace exists, the problem is reduced to finding the right coordinates for the phase. This is straightforward: as stated in Eq. 2.19, real rotations leave scalars invariant and therefore also the scalar component of the tensors considered. Furthermore, the scalar component forms an invariant one-dimensional subspace, which can be directly identified as the subspace onto which the group $\mathbf{P}_{\text {diag }}$ of complex rotations orthogonal to the real rotations must act as a phase. Hence, the separated equation is:

$$
\operatorname{tr}\left[\dot{\varepsilon}_{\text {diag }}\right]=\operatorname{tr}\left[\mathbf{R}^{\mathrm{T}} \dot{\varepsilon}_{\perp} \mathbf{R}+\dot{\varepsilon}_{\|} e^{i \theta}\right]=\operatorname{tr}\left[\mathbf{R}^{\mathbf{T}} \dot{\varepsilon}_{\perp} \mathbf{R}\right]+\operatorname{tr}\left[\dot{\varepsilon}_{\|} e^{i \theta}\right] .
$$

In summary: If real rotations $\mathbf{R}(\mathbf{r})$ are introduced, the actions of $\mathbf{R}(\mathbf{r})$ and $\mathbf{P}(\mathbf{r})$ must be orthogonalized to avoid the introduction of redundant symmetry operations. The group of rotations $\mathbf{P}(\mathbf{r})$ orthogonal to $\mathbf{R}(\mathbf{r})$ then acts only as a phase on the one-dimensional subspace of the scalar components of the strain-rate tensor.

With the orthogonalization, the scalar component can now be treated separately. To introduce the local dependency of the phase, one needs an expression that remains invariant under the global phase transformation; this can be found by squaring the scalar part. One can then write for local phases $\theta(\mathbf{r})$ :

$$
\begin{aligned}
\left(\dot{\varepsilon}_{\|_{\text {diag }}}\right)^{2} & =\left(\boldsymbol{\nabla} \cdot\left(\mathbf{v} e^{-i \theta}\right)\right)\left(\boldsymbol{\nabla} \cdot\left(\mathbf{v}^{\dagger} e^{i \theta}\right)\right) \\
& =\left(\boldsymbol{\nabla} \cdot(\mathbf{v}) e^{-i \theta}-i \mathbf{v} \boldsymbol{\nabla}(\theta) e^{-i \theta}\right)\left(\boldsymbol{\nabla} \cdot\left(\mathbf{v}^{\dagger}\right) e^{i \theta}+i \mathbf{v}^{\dagger} \boldsymbol{\nabla}(\theta) e^{i \theta}\right) \\
& =(\boldsymbol{\nabla} \cdot \mathbf{v}-i \mathbf{v} \boldsymbol{\nabla} \theta)\left(\boldsymbol{\nabla} \cdot \mathbf{v}^{\dagger}+i \mathbf{v}^{\dagger} \boldsymbol{\nabla} \theta\right) \\
& =\left(\boldsymbol{\nabla} \cdot \mathbf{v}^{\dagger}+i \mathbf{v}^{\dagger} \boldsymbol{\nabla} \theta\right)^{2} \\
& =\left(\boldsymbol{\nabla} \cdot \mathbf{v}^{\dagger}+i \mathbf{v}^{\dagger} \mathbf{A}_{\|}\right)^{2}
\end{aligned}
$$

for which the definition $\mathbf{A}_{\|}=\nabla \theta(\mathbf{r})$ was used in the last step.

This results in two complex conjugated roots for the scalar part of the strain-rate tensor, whereby in the following only one root is evaluated first (the second root and implications caused by squaring are dealt with in the later sections). In continuation:

$$
\begin{aligned}
\dot{\varepsilon_{\|_{\text {diag }}}} & =\boldsymbol{\nabla} \cdot \mathbf{v}^{\dagger}+i \mathbf{v}^{\dagger} \mathbf{A}_{\|} \\
& =\operatorname{tr}\left[\dot{\varepsilon}_{\|_{\text {local }}}(\mathbf{r})\right]+\operatorname{tr}\left[\dot{\varepsilon}_{\|_{\text {connect. }} \mathbf{U}(\mathbf{1})}(\mathbf{r})\right],
\end{aligned}
$$

which again can be reordered to:

$$
\operatorname{tr}\left[\dot{\varepsilon}_{\|_{\text {local }}}(\mathbf{r})\right]=\operatorname{tr}\left[\dot{\varepsilon}_{\|_{\text {diag }}}(\mathbf{r})\right]-\operatorname{tr}\left[\varepsilon_{\|_{\text {connect. U(1) }}}(\mathbf{r})\right] .
$$

According to Eqs. 2.20 and 2.25, this finally results in the trace of the strain-rate tensor being:

$$
\begin{aligned}
\operatorname{tr}\left[\dot{\varepsilon}_{\text {local }}(\mathbf{r})\right] & =\operatorname{tr}\left[\dot{\varepsilon}_{\text {diag }}(\mathbf{r})\right]-\operatorname{tr}\left[\dot{\varepsilon}_{\perp \text { connect. SO(3) }}(\mathbf{r})\right]-\operatorname{tr}\left[\dot{\varepsilon}_{\|_{\text {connect. U(1) }}}(\mathbf{r})\right] \\
& =\operatorname{tr}\left[\dot{\varepsilon}_{\operatorname{diag}}(\mathbf{r})\right]-\operatorname{tr}\left[i \mathbf{A}_{\perp}^{\mathbf{a}} \mathbf{v}^{\dagger}\right]-\operatorname{tr}\left[i \mathbf{A}_{\|} \mathbf{v}^{\dagger}\right] .
\end{aligned}
$$

Where $\dot{\varepsilon}_{\text {diag }}$ is as in Eq. 2.1.

\subsubsection{Full strain-rate tensor}

In order to obtain the full dynamics of the gauge fields, their kinetic terms are introduced. For this purpose the gauge fields $\mathbf{A}_{\|}$and $\mathbf{A}_{\perp}^{\mathbf{a}}$ are written as vector potentials 
8

T. Merz

(Sanchez-Monroy \& Quimbay 2010):

$$
\begin{aligned}
& \mathbf{B}_{\|}(\mathbf{r})=\nabla \times \mathbf{A}_{\|} \\
& \mathbf{B}_{\perp}^{\mathbf{a}}(\mathbf{r})=\nabla \times \mathbf{A}_{\perp}^{\mathbf{a}}-\frac{1}{2} \mathbf{f}_{\mathbf{a b c}}\left(\mathbf{A}_{\perp}^{\mathbf{b}} \times \mathbf{A}_{\perp}^{\mathbf{c}}\right) .
\end{aligned}
$$

With the help of these fields, respective field strength tensors can be defined:

$$
\begin{aligned}
F_{j k} & =-\varepsilon_{i j k}\left(B_{\|}\right)^{i} \\
G_{j k}^{a} & =-\varepsilon_{i j k}\left(B_{\perp}^{a}\right)^{i} .
\end{aligned}
$$

$\mathbf{B}_{\|}(\mathbf{r})$ and $\mathbf{B}_{\perp}^{\mathbf{a}}(\mathbf{r})$ are axial vector fields and $\mathbf{B}_{\perp}^{\mathbf{a}}(\mathbf{r})$ has bivectors as components. $\mathbf{F}_{\mathbf{j k}}(\mathbf{r})$ and $\mathbf{G}_{\mathbf{j k}}^{\mathbf{a}}(\mathbf{r})$ are the respective bivector fields associated with the axial vector fields.

The inherent dynamics of the gauge fields can be expressed in their covariant form as a function of the field strength tensors and can then be incorporated into the trace of the strain-rate tensor as follows:

$$
\begin{aligned}
\operatorname{tr}\left[\dot{\varepsilon}_{\text {local }}\right] & =\operatorname{tr}\left[\dot{\varepsilon}_{\text {diag }}-\dot{\varepsilon}_{\text {connect. }}(1)-\dot{\varepsilon}_{\text {connect. SO(3) }}+\dot{\varepsilon}_{\mathbf{U}(1)}+\dot{\varepsilon}_{\mathbf{S O}(3)}\right] \\
& =\operatorname{tr}\left[\dot{\varepsilon}_{\text {diag }}-i \mathbf{A}_{\|} \mathbf{v}^{\dagger}-i \mathbf{A}_{\perp}^{\mathbf{a}} \mathbf{v}^{\dagger}\right]-\frac{1}{4} \mathbf{F}_{\mathbf{j k}} \mathbf{F}^{\mathbf{j k}}-\frac{1}{2} \mathbf{G}^{\mathbf{a}}{ }_{\mathbf{j k}} \mathbf{G}^{\mathbf{a j k}}
\end{aligned}
$$

The kinetic terms of the gauge fields can be rewritten such that they would be included in the trace, applying the anti-symmetry of the field strength tensors:

$$
\begin{aligned}
-\frac{1}{4} F_{j k} F^{j k} & =+\frac{1}{4} F_{j}{ }^{k} F_{k}{ }^{j}=\frac{1}{2} F_{j}{ }^{k} F_{k}{ }^{l} \delta_{l}{ }^{j}-\frac{1}{4} F_{j}{ }^{k} F_{k}{ }^{j} \\
-\frac{1}{2} G^{a}{ }_{j k} G^{a j k} & =+\frac{1}{2} G^{a}{ }_{j}{ }^{k} G^{a}{ }_{k}{ }^{j}=G^{a}{ }_{j}{ }^{k} G^{a}{ }_{k}{ }^{l} \delta_{l}{ }^{j}-\frac{1}{2} G^{a{ }^{k}{ }^{k} G^{a}{ }_{k}{ }^{j} .}
\end{aligned}
$$

In the last step, the degree of freedom to add trace-free linear combinations of the terms was used, leaving the trace invariant. In vectorial notation this becomes:

$$
\begin{aligned}
\operatorname{tr}\left[\dot{\varepsilon}_{\mathbf{U}(\mathbf{1})}\right] & =-\frac{1}{4} \mathbf{F}_{\mathbf{j k}} \mathbf{F}^{\mathbf{j k}}=+\frac{1}{4} \operatorname{tr}\left(\mathbf{F}^{2}\right)=\operatorname{tr}\left[\frac{1}{2} \mathbf{F}^{2}-\frac{1}{4} \operatorname{tr}\left(\mathbf{F}^{2}\right)\right] \\
\operatorname{tr}\left[\dot{\boldsymbol{\varepsilon}}_{\mathbf{S O}(\mathbf{3})}\right] & =-\frac{1}{2} \mathbf{G}^{\mathbf{a}} \mathbf{j k}_{\mathbf{k}} \mathbf{G}^{\mathbf{a} \mathbf{j k}}=+\frac{1}{2} \operatorname{tr}\left(\left(\mathbf{G}^{\mathbf{a}}\right)^{2}\right)=\operatorname{tr}\left[\left(\mathbf{G}^{\mathbf{a}}\right)^{2}-\frac{1}{2} \operatorname{tr}\left(\left(\mathbf{G}^{\mathbf{a}}\right)^{2}\right)\right] .
\end{aligned}
$$

In this form, the outer trace can be removed on both sides of the equation, to obtain the strain-rate tensor (determined up to a similarity transformation):

$$
\dot{\varepsilon}_{\text {local }}=\dot{\varepsilon}_{\text {diag }}-i \mathbf{A}_{\|} \mathbf{v}^{\dagger}-i \mathbf{A}_{\perp}^{\mathbf{a}} \mathbf{v}^{\dagger}+\frac{1}{2} \mathbf{F}^{2}-\frac{1}{4} \operatorname{tr}\left(\mathbf{F}^{2}\right)+\left(\mathbf{G}^{\mathbf{a}}\right)^{2}-\frac{1}{2} \operatorname{tr}\left(\left(\mathbf{G}^{\mathbf{a}}\right)^{2}\right) .
$$

Where $\dot{\varepsilon}_{\text {diag }}$ is as in Eq. 2.1.

\subsection{Gauge invariant stress-tensor}

The relation between stress-tensor and strain-rate tensor is expressed by the constitutive equation Eq. 1.5. Thus, the local stress tensor according to Eq. 2.32 can be inputted 
to obtain:

$$
\begin{aligned}
\sigma_{\text {local }}= & 2 \mu \dot{\varepsilon}_{\perp \text { local }}+3 \zeta \dot{\varepsilon}_{\| \text {local }} \\
= & \sigma_{\perp \text { diag }}+\sigma_{\| \text {diag }}-\sigma_{\text {connect. SO }(3)}-\sigma_{\text {connect. } \mathbf{U}(\mathbf{1})}+\sigma_{\mathbf{S O}(\mathbf{3})}+\sigma_{\mathbf{U}(\mathbf{1})} \\
= & 2 \mu \dot{\varepsilon}_{\text {diag }}+\left(\zeta-\frac{2 \mu}{3}\right)(\nabla \cdot \mathbf{v}) \mathbb{1} \\
& -2 \mu i \mathbf{v}^{\dagger} \mathbf{A}_{\perp}^{\mathbf{a}}-3 \zeta i \mathbf{v}^{\dagger} \mathbf{A}_{\|} \\
& +2 \mu\left[\left(\mathbf{G}^{\mathbf{a}}\right)^{2}-\frac{1}{2} \operatorname{tr}\left(\left(\mathbf{G}^{\mathbf{a}}\right)^{2}\right)\right]+3 \zeta\left[\frac{1}{2} \mathbf{F}^{2}-\frac{1}{4} \operatorname{tr}\left(\mathbf{F}^{2}\right)\right]
\end{aligned}
$$

\subsection{Identification of gauge fields}

To apply these results to the Cauchy momentum equations Eq. 1.4, it is necessary to calculate the divergence of the stress tensor from Eq. 2.33. For this purpose, the individual components will be considered:

Divergence of the diffusion component: The diffusion component $\boldsymbol{\sigma}_{\text {diag }}$ is already diagonalized, such that the divergence can be expressed as:

$$
\boldsymbol{\nabla} \cdot \boldsymbol{\sigma}_{\text {diag }}=2 \mu \nabla \cdot \dot{\varepsilon}_{\text {diag }}+\left(\zeta-\frac{2 \mu}{3}\right) \nabla(\nabla \cdot \mathbf{v}) .
$$

Where it was assumed that, within the region of interest, the viscosities $\mu$ and $\zeta$ are approximately constant ( $\dot{\varepsilon}_{\text {diag }}$ as in Eq. 2.1).

To simplify this expression somewhat further, it can be used that $\dot{\varepsilon}_{\text {diag }}$ is rotation-free $(\boldsymbol{\nabla} \times \mathbf{v}=0)$, since its off-diagonal components are zero everywhere $\dot{\varepsilon}_{\text {diag }}{ }_{k}{ }_{k}=\partial^{j} v_{k}^{\dagger}=0$ for $j \neq k$, and its eigenvalues are real $\dot{\varepsilon}_{\text {diag }}{ }_{k}{ }_{k}=\delta_{k}^{j} \partial^{j} v_{k}^{\dagger}=\delta_{k}^{j} \partial^{j} v$ :

$$
\begin{aligned}
\boldsymbol{\nabla} \cdot \boldsymbol{\sigma}_{\text {diag }} & =2 \mu \boldsymbol{\nabla} \cdot \dot{\varepsilon}_{\text {diag }}+\left(\zeta-\frac{2 \mu}{3}\right) \boldsymbol{\nabla}(\boldsymbol{\nabla} \cdot \mathbf{v}) \\
& =2 \mu \nabla^{2} \mathbf{v}+\left(\zeta-\frac{2 \mu}{3}\right) \nabla^{2} \mathbf{v}+\boldsymbol{\nabla} \times(\boldsymbol{\nabla} \times \mathbf{v}) \\
& =\left(\zeta+\frac{4 \mu}{3}\right) \nabla^{2} \mathbf{v} .
\end{aligned}
$$

Divergence of the interaction terms: The divergence of the interaction terms disappears if the gauging of the fields is chosen to be $\boldsymbol{\nabla} \cdot \mathbf{A}_{\|}=0$ and $\boldsymbol{\nabla} \cdot \mathbf{A}_{\perp}^{\mathbf{a}}=0$, since, by additionally using the continuity equation Eq. 1.9 , one has:

$$
\begin{aligned}
\boldsymbol{\nabla} \cdot \boldsymbol{\sigma}_{\text {connection }}= & -\boldsymbol{\nabla} \cdot\left(2 \mu i \mathbf{v}^{\dagger} \mathbf{A}_{\perp}^{\mathbf{a}}\right)-\nabla \cdot\left(3 \zeta i \mathbf{v}^{\dagger} \mathbf{A}_{\|}\right) \\
= & -\nabla \cdot\left(2 R e^{-1} \rho i \mathbf{v}^{\dagger} \mathbf{A}_{\perp}^{\mathbf{a}}\right)-\nabla \cdot\left(3 \frac{\zeta^{x}}{\mu^{x}} R e^{-1} \rho i \mathbf{v}^{\dagger} \mathbf{A}_{\|}\right) \\
= & -2 R e^{-1} i\left(\nabla \cdot\left(\rho \mathbf{v}^{\dagger}\right) \mathbf{A}_{\perp}^{\mathbf{a}}-\rho \mathbf{v}^{\dagger} \nabla \cdot\left(\mathbf{A}_{\perp}^{\mathbf{a}}\right)\right) \\
& -3 \frac{\zeta^{x}}{\mu^{x}} R e^{-1} i\left(\nabla \cdot\left(\rho \mathbf{v}^{\dagger}\right) \mathbf{A}_{\|}-\rho \mathbf{v}^{\dagger} \nabla \cdot\left(\mathbf{A}_{\|}\right)\right) \\
= & 0 .
\end{aligned}
$$

In this step, the Reynolds number $R e$ and the ratio $\frac{\zeta^{x}}{\mu^{x}}$ between dynamic and volume viscosity are treated as approximately constant within the region of interest.

Divergence of the kinetic gauge field terms: What remains are the kinetic terms 
10

\section{T. Merz}

of the gauge fields, whose components are considered in the following:

$$
\begin{aligned}
\left(\sigma_{U(1)}\right)_{j}{ }^{l} & =\frac{3 \zeta}{2} F_{j}{ }^{k} F_{k}{ }^{l}-\frac{3 \zeta}{4} F_{j}{ }^{k} F_{k}{ }^{j} \\
& =\frac{3 \zeta}{2} \varepsilon_{i j k} \varepsilon^{m k l} B_{\|}^{i} B_{\| m}-\frac{3 \zeta}{4} \varepsilon_{i j k} \varepsilon^{n k j} B_{\|}^{i} B_{\| n} \\
& =\frac{3 \zeta}{2} \varepsilon_{k i j} \varepsilon^{k l m} B_{\|}^{i} B_{\| m}+\frac{3 \zeta}{4} \varepsilon_{i j k} \varepsilon^{n j k} B_{\|}^{i} B_{\| n} \\
& =\frac{3 \zeta}{2}\left(\delta_{i}^{l} \delta_{j}^{m}-\delta_{i}^{m} \delta_{j}^{l}\right) B_{\|}^{i} B_{\| m}+\frac{3 \zeta}{4} 2 \delta_{i}^{n} B_{\|}^{i} B_{\| n} \\
& =\frac{3 \zeta}{2} B_{\|}^{i} B_{\| j}-\frac{3 \zeta}{2} B_{\|}^{i} B_{\| i}+\frac{3 \zeta}{2} B_{\|}^{i} B_{\| i} \\
& =\frac{3 \zeta}{2} B_{\|}^{i} B_{\| j}
\end{aligned}
$$

as well as

$$
\begin{aligned}
\left(\sigma_{S O(3)}\right)_{j}{ }^{l} & =2 \mu G^{a}{ }_{j}{ }^{k} G^{a}{ }_{k}{ }^{l}-2 \mu \frac{1}{2} G^{a}{ }_{j}{ }^{k} G^{a}{ }_{k}{ }^{j} \\
& =2 \mu \varepsilon_{i j k} \varepsilon^{m k l} B_{\perp}^{a i} B_{\perp m}^{a}-2 \mu \frac{1}{2} \varepsilon_{i j k} \varepsilon^{n k j} B_{\perp}^{a i} B_{\perp n}^{a} \\
& =2 \mu \varepsilon_{k i j} \varepsilon^{k l m} B_{\perp}^{a i} B_{\perp m}^{a}+2 \mu \frac{1}{2} \varepsilon_{i j k} \varepsilon^{n j k} B_{\perp}^{a i} B_{\perp n}^{a} \\
& =2 \mu\left(\delta_{i}^{l} \delta_{j}^{m}-\delta_{i}^{m} \delta_{j}^{l}\right) B_{\perp}^{a i} B_{\perp m}^{a}+2 \mu \frac{1}{2} 2 \delta_{i}^{n} B_{\perp}^{a i} B_{\perp n}^{a} \\
& =2 \mu B_{\perp}^{a i} B_{\perp j}^{a}-2 \mu B_{\perp}^{a i} B_{\perp i}^{a}+2 \mu B_{\perp}^{a i} B_{\perp i}^{a} \\
& =2 \mu B_{\perp}^{a i} B_{\perp j}^{a} .
\end{aligned}
$$

The anti-symmetry of the Levi-Civita symbol under index permutation, as well as its relations to the Kronecker-Delta $\varepsilon_{k i j} \varepsilon^{k l m}=\left(\delta_{i}^{l} \delta_{j}^{m}-\delta_{i}^{m} \delta_{j}^{l}\right)$ and $\varepsilon_{i j k} \varepsilon^{n j k}=2 \delta_{i}^{n}$ were used in this calculation.

In vectorial notation this becomes:

$$
\boldsymbol{\sigma}_{\mathbf{S O}(3)}=2 \mu \mathbf{B}_{\perp}^{\mathbf{a}} \otimes \mathbf{B}_{\perp}^{\mathbf{a}}=\rho\left(2 R e^{-1} \mathbf{B}_{\perp}^{\mathbf{a}} \otimes \mathbf{B}_{\perp}^{\mathbf{a}}\right)=: \rho\left(\mathbf{v}_{\perp}^{\mathbf{a}} \otimes \mathbf{v}_{\perp}^{\mathbf{a}}\right)
$$

and

$$
\boldsymbol{\sigma}_{\mathbf{U}(\mathbf{1})}=\frac{3 \zeta}{2} \mathbf{B}_{\|} \otimes \mathbf{B}_{\|}=\frac{\rho}{2}\left(\frac{3 \zeta^{x}}{\mu^{x}} R e^{-1} \mathbf{B}_{\|} \otimes \mathbf{B}_{\|}\right)=: \frac{\rho}{2}\left(\mathbf{v}_{\|} \otimes \mathbf{v}_{\|}\right) .
$$

In the respective last steps, the following scaled gauge fields were defined:

$$
\mathbf{v}_{\perp}^{\mathbf{a}}=\sqrt{2 R e^{-1}} \mathbf{B}_{\perp}^{\mathbf{a}} \quad \text { and } \quad \mathbf{v}_{\|}=\sqrt{\frac{3 \zeta^{x}}{\mu^{x}} R e^{-1}} \mathbf{B}_{\|} .
$$

Next, the divergence is calculated, taking advantage of the fact that by construction the $\mathbf{v}_{\perp}^{\mathbf{a}}$ are orthogonal to $\mathbf{v}_{\|}$(e.g. Huybrechts (2004)):

$$
\begin{aligned}
\nabla \cdot\left(\boldsymbol{\sigma}_{\mathbf{S O}(3)}+\sigma_{\mathbf{U}(1)}\right) & =\nabla \cdot\left(2 \mu \dot{\varepsilon}_{\mathbf{S O}(3)}+3 \zeta \dot{\varepsilon}_{\mathbf{U}(\mathbf{1})}\right) \\
& =\nabla \cdot\left[\rho\left(\mathbf{v}_{\perp}^{\mathbf{a}} \otimes \mathbf{v}_{\perp}^{\mathbf{a}}+\frac{1}{2} \mathbf{v}_{\|} \otimes \mathbf{v}_{\|}\right)\right] \\
& =\nabla \cdot\left[\rho\left(\mathbf{v}_{\perp}^{\mathbf{a}}+\frac{1}{\sqrt{2}} \mathbf{v}_{\|}\right) \otimes\left(\mathbf{v}_{\perp}^{\mathbf{a}}+\frac{1}{\sqrt{2}} \mathbf{v}_{\|}\right)\right] .
\end{aligned}
$$

This expression has the same form as the convection term of the Navier-Stokes equations 
in their conservation form, Eq. 1.10 .

$$
\nabla \cdot(\rho \mathbf{v} \otimes \mathbf{v}) .
$$

Furthermore, the gauge field components $\mathbf{v}_{\perp}^{\mathbf{a}}$ and $\mathbf{v}_{\|}$from Eq. 2.41 transform like axial vectors, and thus, up to space inversion, the same as the velocity field $\mathbf{v}$ in Eq. 2.43.

The issue of space inversion is addressed in the next paragraph, and solved by changing the sign of the coupling constant when inverting space.

With this, the scaled gauge fields $\mathbf{v}_{\perp}^{\mathbf{a}}$ and $\mathbf{v}_{\|}$agree both in form and transformation properties with the convection term of the Navier-Stokes equations and can therefore be identified with each other. According to Eq. 2.42 and Eq. 2.43 the identification reads:

$$
\mathbf{v}=\mathbf{v}_{\perp}^{\mathbf{a}}+\frac{1}{\sqrt{2}} \mathbf{v}_{\|} .
$$

The $\frac{1}{\sqrt{2}}$-pre-factor is present due to the fact that $\mathbf{v}_{\|}$is abelian.

Divergence of the overall stress tensor: Hence, the divergence of the stress tensor becomes in summary:

$$
\begin{aligned}
& \nabla \cdot \sigma_{\text {local }}=\nabla \cdot \sigma_{\text {diag }}+\nabla \cdot \sigma_{\text {connection }}+\nabla \cdot\left(\sigma_{\mathrm{SO}(3)}+\sigma_{\mathrm{U}(1)}\right) \\
& =2 \mu \nabla \cdot \dot{\varepsilon}_{\text {diag }}+\left(\zeta-\frac{2 \mu}{3}\right) \nabla(\nabla \cdot \mathbf{v})+\nabla \cdot(\rho \mathbf{v} \otimes \mathbf{v}) \\
& =\left(\zeta+\frac{4 \mu}{3}\right) \nabla^{2} \mathbf{v}+\nabla \cdot(\rho \mathbf{v} \otimes \mathbf{v}) \text {. }
\end{aligned}
$$

The constructed stress tensor $\boldsymbol{\sigma}_{\text {local }}$ thus already contains the convection information, offering several options to reformulate the problem of fluid mechanics:

It can either be used to express the Navier-Stokes equations omitting the nonlinear convective part of the material derivative $\frac{D}{D t}$ (see Eq. 1.4) and replacing it by the partial derivative, since this information is included in $\sigma_{\text {local }}$ :

$$
\rho \frac{\partial \mathbf{v}}{\partial t}=\nabla \cdot \sigma_{\text {local }}-\nabla p+\rho \mathbf{g}
$$

or one represents the internal and external forces as potentials and inserts them directly into the stress tensor, obtaining a system of equations with the full information contained in the stationary Navier-Stokes equations:

$$
\sigma_{\text {total }}=\sigma_{\text {local }}-p+G
$$

where $\boldsymbol{\nabla} \cdot G=\rho \mathbf{g}$.

\subsection{Normalized form}

From the scaled vector fields Eq. 2.41, scaled field tensors and vector potentials can be defined:

$$
\begin{aligned}
F_{j k}^{\prime} & =-\varepsilon_{i j k}\left(v_{\|}\right)^{i}=\sqrt{\frac{3 \zeta^{x}}{\mu^{x} R e}} F_{j k} \\
G_{j k}^{\prime a} & =-\varepsilon_{i j k}\left(v_{\perp}^{a}\right)^{i}=\sqrt{\frac{2}{R e}} G_{j k}^{a}
\end{aligned}
$$




$$
\begin{aligned}
\mathbf{A}_{\|}^{\prime} & =\sqrt{\frac{3 \zeta^{x}}{\mu^{x} R e}} \mathbf{A}_{\|}=\sqrt{3 \zeta \rho^{-1}} \mathbf{A}_{\|} \\
\mathbf{A}_{\perp}^{\prime \mathbf{a}} & =\sqrt{\frac{2}{R e}} \mathbf{A}_{\perp}^{\mathbf{a}}=\sqrt{2 \mu \rho^{-1}} \mathbf{A}_{\perp}^{\mathbf{a}}
\end{aligned}
$$

with

$$
\begin{aligned}
\mathbf{v}_{\|} & =\nabla \times \mathbf{A}_{\|}^{\prime} \\
\mathbf{v}_{\perp}^{\mathbf{a}} & =\nabla \times \mathbf{A}_{\perp}^{\prime \mathbf{a}}-\frac{1}{2} \mathbf{f}_{\mathrm{abc}}\left(\mathbf{A}_{\perp}^{\prime \mathbf{b}} \times \mathbf{A}_{\perp}^{\prime \mathrm{c}}\right) .
\end{aligned}
$$

Going back to the trace of the stress tensor Eq. 2.33 this reads in terms of the scaled fields, using that the trace of a dyadic product can be expressed as a scalar product $\operatorname{tr}\left[\mathbf{a b}^{\dagger}\right]=\mathbf{a} \cdot \mathbf{b}^{*}$, as well as $\sigma_{\operatorname{diag}}{ }_{k}{ }_{k}=\delta_{k}^{j} \partial^{j} v_{k}^{\dagger}=\delta_{k}^{j} \partial^{j} v_{k} \in \mathbb{R}$ :

$$
\begin{aligned}
& \operatorname{tr}\left[\boldsymbol{\sigma}_{\text {local }}\right]=\left(\zeta+\frac{4 \mu}{3}\right) \operatorname{tr}\left[\boldsymbol{\nabla} \mathbf{v}^{\dagger}\right]-2 \mu i \operatorname{tr}\left[\mathbf{A}_{\perp}^{\mathbf{a}} \mathbf{v}^{\dagger}\right]-3 \zeta i \operatorname{tr}\left[\mathbf{A}_{\|} \mathbf{v}^{\dagger}\right]-\mu \mathbf{G}_{\mathbf{j k}}^{\mathbf{a} \mathbf{G}} \mathbf{G}^{\mathbf{a} \mathbf{j k}}-\frac{3 \zeta}{4} \mathbf{F}_{\mathbf{j k}} \mathbf{F}^{\mathbf{j k}} \\
= & \left(\zeta+\frac{4 \mu}{3}\right) \boldsymbol{\nabla} \cdot \mathbf{v}^{*}-\sqrt{\frac{2}{R e}} \rho i \mathbf{A}_{\perp}^{\mathbf{a}} \cdot \mathbf{v}^{*}-\sqrt{\frac{3 \zeta^{x}}{\mu^{x} R e}} \rho i \mathbf{A}_{\|}^{\prime} \cdot \mathbf{v}^{*}-\frac{1}{2} \rho \mathbf{G}^{\prime \mathbf{a}}{ }_{\mathbf{j k}} \mathbf{G}^{\mathbf{a} \mathbf{j k}}-\frac{1}{4} \rho \mathbf{F}_{\mathbf{j k}}^{\prime} \mathbf{F}^{\prime \mathbf{j k}} .
\end{aligned}
$$

This expression can be brought into the standard form of Yang-Mills theories with normalized kinetic terms according to $\frac{1}{2} \mathbf{G}^{\prime \mathbf{a}}{ }_{\mathbf{j k}} \mathbf{G}^{\mathbf{a}}{ }^{\mathbf{j k}}$ and $\frac{1}{4} \mathbf{F}_{\mathbf{j k}}^{\prime} \mathbf{F}^{\prime \mathbf{j k}}$ by dividing through the density:

$$
\rho^{-1} \operatorname{tr}\left[\boldsymbol{\sigma}_{\text {local }}\right]=\left(\frac{\zeta^{x}}{\mu^{x}}+\frac{4}{3}\right) R e^{-1} \mathbf{D} \cdot \mathbf{v}^{*}-\frac{1}{2} \mathbf{G}^{\prime \mathbf{a}}{ }_{\mathbf{j k}} \mathbf{G}^{\prime \mathbf{a} \mathbf{j k}}-\frac{1}{4} \mathbf{F}_{\mathbf{j k}}^{\prime} \mathbf{F}^{\prime \mathbf{j k}} .
$$

Finally defining the gauge covariant derivative $\mathbf{D}$ and the coupling constants $g^{\prime}$ and $g$ between the locally diagonalized diffusion term and the convection fields:

$$
\mathbf{D}=\nabla-g^{\prime} \mathbf{A}_{\perp}^{\prime \mathbf{a}}-g \mathbf{A}_{\|}^{\prime}
$$

with

$$
g^{\prime}=\frac{i \sqrt{2}}{\left(\frac{\zeta^{x}}{\mu^{x}}+\frac{4}{3}\right)} \sqrt{R e} \quad \text { and } \quad g=\frac{i \sqrt{\frac{3 \zeta^{x}}{\mu^{x}}}}{\left(\frac{\zeta^{x}}{\mu^{x}}+\frac{4}{3}\right)} \sqrt{R e} .
$$

Please note that as stated in Section 2.6 the sign of the coupling constants is inverted upon space inversion in order to achieve the correct transformation properties of the underlying gauge fields: $\breve{g}=-g$ and $\breve{g}^{\prime}=-g^{\prime}$.

\section{Conclusion}

A method was presented to locally diagonalize the strain-rate tensor found in fluid dynamics in the entire space, using $S O(3) \times U(1)$ gauge fields. From this, a locally diagonal stress tensor was determined which is coupled through the gauge fields to the surrounding stresses. It was derived that the diagonal component contains the diffusion terms of the flow, whereas the gauge fields contain the convective parts.

In addition, the stress tensor contains interaction terms which describe the interaction between diffusion and convection terms. Taken the material-related pre-factors in the 


\section{Application of local gauge theories to fluid mechanics}

gauge coupling constants as a given, the interaction terms couple proportionally to the square root of the Reynolds number $\sqrt{R e}$ to the diffusion terms (Eq. 2.54), thus exhibiting the expected property that flows behave according to the Reynolds similarity law.

The introduced gauge fields describe the symmetry properties of convective flows to a previously unknown degree: the convection is composed of four antisymmetric flow fields, of which three are volume preserving and one is not. The volume preserving fields are non-abelian and show self-interactions of higher order.

It was shown that the stress tensor constructed according to Eq. 2.47 contains the complete information of the stationary Navier-Stokes equations. This equation can thus be used as an alternative basic equation for stationary problems in fluid dynamics, and offers advantages over the Navier-Stokes equations in terms of interpretation and/or solution approaches, depending on the application.

Since the structure of Eq. 2.52 is very similar to minimal coupling field theories in quantum mechanics, it can be expected, that - with favorable boundary conditions - the gauge fields can be quantized using the second quantization formalism.

This makes it possible to interpret the gauge fields as quasi-particles with properties depending on the Reynolds number. Due to the properties of the underlying symmetry groups, the quasi-particles have bosonic spin 1 character, and thus contain an inherent angular momentum. They can therefore be interpreted as elementary vortices. The $S O(3)$ fields can be understood as elementary vortices with orientation in the three Cartesian spatial directions, which interact with each other. It is interesting to note that in twodimensional flows the self-interaction disappears, because in this case the system of equations is reduced to a $S O(2) \times U(1)$ theory with the abelian rotating group $S O(2)$.

The $U(1)$ convection field interacts due to volume viscosity and disappears in noncompressible fluids.

For weakly coupling fields $(\sqrt{R e} \ll 1)$, it is to be expected that average creation and annihilation probabilities, as well as lifetimes of the elementary vortices can be determined from perturbative calculations, which in turn could be verified experimentally. However, more detailed examinations on this topic must be left for further work.

In strongly coupling flows $(\sqrt{R e}>1)$, which are of particular practial interest, perturbative approaches can not be applied and solutions have to be found numerically. However, the procedure presented may hold some advantages for this case too: the stress tensor in Eq. 2.47 contains all the information of the stationary Navier-Stokes equations, but uses only first order derivatives, which may lead to more stable numerical simulations than second order differentials as in the Navier-Stokes equations.

Outlook The systems of equations presented here can be applied virtually without change to elastic media. They obey the same general mechanics as the Cauchy-momentum equations (Eq. 1.4), but in this case inserting an elastic constitutive equation (roughly $\sigma \propto \varepsilon)$, with $\varepsilon$ the strain tensor. Mathematically this system is equivalent to a fluid, the only significant difference lies in its physical interpretation. Some early versions of these concepts can be found on my private homepage http://elasticuniverse.org in unreviewed form.

The following steps are the next objectives:

- Generalisation of the equations to four dimensions.

- Application of further concepts from field theory, e.g. spontaneous symmetry breaking as phase transition from a fluid to an elastic solid.

- Treatment of elastic media. Introduction of defects. 


\section{Acknowledgments}

My thanks go to Rudolf Fehlmann for the countless, fruitful discussions and in particular for his support in the structuring of my work. Likewise, my thanks go to Benjamin Arner for the translations into English, as well as to Dr. Nelson Bolivar for the pre-publication review. Then to my father for the editing, plus my family and friends for the ongoing support.

Declaration of Interests: None.

\section{REFERENCES}

H. Horner. Turbulenz; Ruprecht-Karls-Universität Heidelberg, Institut für Theoretische Physik: Heidelberg, DE, 2011; Link.

N. Borghini. Theoretische Physik IV; Universität Bielefeld, Fakultät für Physik: Bielefeld, DE, 2013; Link.

B. Urbanek. Turbulente Strömung; Universität Regensburg, Fakultät für Physik: Regensburg, DE, 2012; Link.

N. Coleman, A Derivation of the Navier-Stokes Equations. Ball State Undergraduate Mathematics Exchange 2010, 7, 1. Link

A. Sulaiman. Construction of Navier-Stokes Equation using Gauge Field Theory Approach; Universitas Indonesia: Depok, IDN, 2005; Link.

A. Sulaiman and L.T. Handoko, Lagrangian dynamics of the Navier-Stokes equation. arXiv:physics/0508092 [physics.flu-dyn] 2005. Link

T. H. Moulden, On gauge fields in fluid turbulence. WIT Transactions on Engineering Sciences 2012, 74, 73-84. CrossRef

W. E and J. Liu, Gauge Method for Viscous Incompressible Flows. Communications in Mathematical Sciences 2003, 1 (2), 317-332. CrossRef

P. Constantin. An Eulerian-Lagrangian approach to the Navier-Stokes equations; University of Chicago, Department of Mathematics: Chicago, IL, 2000; Link.

J. Iliopoulos. Introduction to the Standard Model of the Electro-Weak Interactions; CERN Summer School of Particle Physics: Angers, FR, 2012; Link.

J.A. Sanchez-Monroy and C.J. Quimbay, Some classical properties of the non-abelian Yang-Mills theories. Revista Mexicana de Fisica E 2010, 56 (2), 172-176. Link

D. Huybrechts. Complex Geometry - an Introduction; Springer, 2004; ISBN 3-540-21290-6. 